\title{
Photonic microwave and RF programmable true time delays for phased array antennas using a soliton crystal Kerr micro-comb source
}

David Moss ( $\sim$ dmoss@swin.edu.au )

Swinburne University of Technology

\section{Research Article}

Keywords: radio frequency, microwave, true time delay, nonlinear optics, microcombs, Kerr combs, microwave photonics

Posted Date: October 4th, 2021

DOI: https://doi.org/10.21203/rs.3.rs-953859/v1

License: (c) (i) This work is licensed under a Creative Commons Attribution 4.0 International License.

Read Full License 


\section{Abstract}

We demonstrate significantly improved performance of a microwave true time delay line based on an integrated optical frequency comb source. The broadband micro-comb (over $100 \mathrm{~nm}$ wide) features a record low free spectral range of $49 \mathrm{GHz}$, resulting in an unprecedented record high channel number (81 over the $\mathrm{C}$ band) - the highest number of channels for an integrated comb source used for microwave signal processing. We theoretically analyze the performance of a phased array antenna and show that this large channel count results in a high angular resolution and wide beam steering tunable range. This demonstrates the feasibility of our approach as a competitive solution towards implementing integrated photonic true time delays in radar and communications systems.

\section{Introduction}

In modern radar and communications systems, photonic signal processing has attracted great interest due to its numerous intrinsic advantages such as broad operation bandwidth, low loss, and strong immunity to electromagnetic interference [1-5]. Photonic microwave true time delay lines (TTDLs), which can introduce multiple progressive time delays, are one of the basic building blocks of microwave photonic systems. Offering intrinsically low loss, ultra-wide operation bandwidth, greatly reduced footprint, and immunity to electromagnetic interference, photonic TTDLs have wide applications for phased array antennas (PAAs), microwave photonic filters, analog-to-digital or digital-to-analog conversion, as well as arbitrary waveform generation [6-21].

Extensive effort has been devoted to developing diverse methods for TTDLs, including using: dispersive elements such as single-mode fibre (SMF) [22], dispersion compensation fibre [23], fibre Bragg gratings (FBGs) [24-25]; slow-light devices based on stimulated Brillouin scattering (SBS); integrated resonators [26-28]; wavelength conversion coupled with chromatic dispersion [29], and more [30-31]. Further, tunable progressive TTDLs based on a switch-controlled dispersive recirculating loop [32], fast sweeping lasers [33], and dispersion-tunable media [34] have also been investigated.

For PAAs, the number of radiating elements determines the beam-width, and so, in order to enhance the angular resolution, a large channel number is required for the TTDL. Typically, discrete lasers arrays [26, 32-34] or FBG arrays [24-25] have been employed for multiple TTDL channels, resulting in a significant increase in system cost and complexity. In turn, this greatly limits the number of channels in practical systems. Other schemes based on optical frequency combs (OFCs) [6] can mitigate this problem, yet many approaches to generating OFCs, such as those based on cascaded electro-optical (EO) modulators [9,35-40] and Fabry-Perot EO modulators [41], require external radio frequency (RF) sources, which still impose considerable cost and complexity to the TTDL.

On the other hand, OFCs generated by high-Q micro-resonators, termed micro-combs or Kerr combs [4247], offer a new generation of compact, low-cost, and highly efficient multi-wavelength sources, thus bringing about huge possibilities towards achieving high-performance TTDLs. Their advantages include 
the potential for a large number of channels (wavelengths) and greatly reduced footprint and complexity, resulting in significantly improved performance for delay-line structure-based microwave photonic systems.

Recently, $[48,49]$ we demonstrated photonic microwave TTDLs based on an integrated micro-comb source with a free spectral range (FSR) of $200 \mathrm{GHz}$, achieving high performance when applied to PAAs in terms of beam resolution and angular tuning range. In this paper, we significantly improve the performance of this device, both in terms of angular resolution and beamwidth, by employing a microcomb source with a $49 \mathrm{GHz} F S R$, thus dramatically increasing the available number of-channels from 21 to 81 over the C-band, which is a record high for integrated comb source-based TTDLs. We experimentally demonstrate an 81-channel true time delay and then calculate the key performance parameters for a phased array antenna based on our device, thus verifying the feasibility and effectiveness of our approach.

\section{Operation Principle}

Figure 1 shows a schematic of the microwave TTDL based on an integrated optical Kerr comb source. The TTDL contains two modules: the first generates the optical micro-comb using an integrated MRR while the second creates replicas of input RF signals at each wavelength followed by weighting and wavelength dependent time delays (i.e., induced by the dispersive medium) using standard optical fibres to form the high-channel count TTDL for the phased array antenna.

The MRR used to generate the Kerr optical comb (Fig. 2(a)) was fabricated on a high-index doped silica glass platform using CMOS compatible processes [42]. First, high-index ( $\mathrm{n}=\sim 1.7$ at $1550 \mathrm{~nm}$ ) doped silica glass films were deposited using plasma enhanced chemical vapour deposition (PECVD), and then patterned by deep UV photolithography and subsequently processed via reactive ion etching to form waveguides with exceptionally low surface roughness. Finally, silica $(n=\sim 1.44$ at $1550 \mathrm{~nm}$ ) was deposited via PECVD as an upper cladding. Our device architecture used a vertical coupling scheme where the gap can be controlled via film growth - a more accurate approach than lithographic techniques [50]. Other advantages of our platform for nonlinear optics include ultra-low linear loss $(\sim 0.06$ $\left.\mathrm{dB} \llbracket \mathrm{cm}^{-1}\right)$, a relatively high nonlinear parameter $\left(\sim 233 \mathrm{~W}^{-1} \llbracket \mathrm{km}^{-1}\right)$ and, in particular, negligible nonlinear loss up to extremely high intensities $\left(\sim 25 \mathrm{GW} \llbracket \mathrm{cm}^{-2}\right)$ [42-44]. The compact integrated MRR had a radius of $\sim 592 \mu \mathrm{m}$ with a free spectral range (FSR) of $\sim 0.4 \mathrm{~nm}$, i.e., $\sim 49 \mathrm{GHz}$, as indicated in Fig. 2(b) and Fig. 2(d). Although micro-combs with smaller FSRs have been reported with whispering-gallery-mode resonators or SiN micro-ring resonators [51, 52], our device has the smallest FSR of any micro-comb source that has been used for microwave signal processing. This FSR - a factor of 4 smaller than our previous results $[48,49]$ - enabled up to four times the number of channels (as many as 81 channels) over the C-band. After packaging the device with fibre pigtails, the total insertion loss was $\sim 1 \mathrm{~dB}$. Due to the ultra-low propagation loss of our platform, the MRR featured a narrow resonance linewidth (Fig. 2(c)) corresponding to a $\mathrm{Q}$ factor of $\sim 1.2$ million. In order to obtain optimal parametric gain, the MRR was designed to feature anomalous dispersion in the C-band [53]. 
In the first module, CW pump light from a tunable laser source was amplified by an erbium-doped fibre amplifier (EDFA) to pump the on-chip MRR. Before the MRR, a tunable optical bandpass filter and a polarization controller were employed to suppress the amplified spontaneous emission noise and adjust the polarization state, respectively. When the pump wavelength was tuned to one of the resonances of the MRR and the pump power was high enough to provide sufficient parametric gain, parametric oscillation in the MRR occurred, ultimately generating a Kerr optical comb with a high degree of equal line spacing [42-43]. In order to avoid resonance drift and maintain the wavelength alignment of the resonances to the pump light, the MRR was mounted on a temperature controlled stage.

In the second module, the generated Kerr comb was directed to a Mach-Zehnder modulator (MZM), where replicas of an input RF signal were produced at each wavelength. The output optical signals from the MZM were delayed by a dispersive SMF, yielding a time delay difference between adjacent channels. Finally, individual channels were manipulated by a waveshaper, separated by a wavelength division multiplexer (WDM), thus achieving a multichannel RF TTDL based on Kerr combs.

We note that integrated dispersive delays for fully integrated beamforming systems have been achieved recently using promising approaches, including chirped Bragg gratings [54], photonic crystals [55], and low loss silicon nitride waveguides [56].

In PAA systems, optical signals on selected TTDL channels are separately converted into the electrical domain and then sent to an antenna array to drive the radiating elements. Considering that the antenna array is a uniformly spaced linear array with an element spacing of $d_{P A A}$, the steering angle $\theta_{0}$ of the PAA can be given as [57]

$$
\theta_{0}=\sin ^{-1} \frac{c \cdot \tau}{d_{P A A}}
$$

where $c$ is the speed of light in vacuum, and $\tau$ is the time delay difference between adjacent radiating elements. From Eq. (1), one can see that the steering angle can be tuned by adjusting $\tau$, i.e., changing the length of the dispersive medium or simply selecting every $m_{t h}(m=1,2,3, \ldots)$ TTDL channel as radiating elements. These two approaches for tuning $\tau$ offer complementary advantages - either fine tuning steps or a large tuning range, respectively - and so they can be combined to achieve optimized performance for practical applications. In the latter case, $\tau=m T$, where $T$ is the time delay difference between adjacent channels, which is determined jointly by the frequency spacing of the comb source and the dispersion accumulated in the delay line. Thus, the steering angle is given by

$$
\theta_{0}=\sin ^{-1} \frac{c \cdot m T}{d_{P A A}}
$$

And the corresponding array factor (AF) of the PAA can be expressed as [57] 


$$
\mathrm{G} A F\left(\theta, \lambda_{R F}\right)=\frac{\sin ^{2}\left[M \pi\left(d_{P A A} / \lambda_{R F}\right)\left(\sin \theta-c \cdot m T / d_{P A A}\right)\right]}{M^{2} \sin ^{2}\left[\pi\left(d_{P A A} / \lambda_{R F}\right)\left(\sin \theta-c \cdot m T / d_{P A A}\right)\right]}
$$

where $\theta$ is the radiation angle, $M$ is the number of radiating elements, and $\lambda_{R F}$ is the wavelength of the RF signals. The angular resolution of the PAA is the minimum angular separation at which two equal targets at the same range can be separated, and is determined by the 3-dB beam-width that can be approximated [58] as $\theta_{3 d B}=102 / M$, which in turn greatly decreases with the number of radiating elements (M). Our TTDL, based on an integrated optical comb source, provides a large number of radiating elements for beam steering, resulting in a greatly enhanced angular resolution of the PAA. Compared with existing techniques based on discrete laser diode arrays, our approach features a compact and simplified structure, with potentially significantly reduced cost and high performance brought about by the large number of delay channels.

\section{Experimental And Theoretical Results}

In order to generate the $49 \mathrm{GHz}$ spacing micro-comb, the pump power was boosted up to $30.5 \mathrm{dBm}$ via an EDFA and the wavelength was swept towards the red side. When the detuning between pump wavelength and MRR's resonance became small enough such that the intracavity field reached a threshold value, modulation instability was initiated, and primary combs arose with a spacing of multiple FSRs. As the detuning was further changed, the parametric gain lobes broadened and secondary comb lines with a spacing equal to the FSR of the MRR were generated by both degenerate and non-degenerate four wave mixing. This finally resulted in a single FSR spaced Kerr optical comb [44, 59] (Fig. 2(e)) that was over 100nm wide. A zoom-in view (Fig. 2(f)) indicates that potentially over 400 wavelengths were available for the TTDL. For our experiments, however, the wavelength range was limited to the $\mathrm{C}$-band because of the waveshaper and EDFA, and so this resulted in 81 usable channels, or wavelengths.

While the spectral profile of our comb was not indicative of operation in the single cavity soliton regime, at the same time the observed stability of the comb spectrum made it clear that we were not operating in the chaotic regime of the Ikeda map $[60,61]$. In fact, both the shape and stability of our comb, we believe, are indicative of possible operation in multiple soliton states such as recently reported soliton crystals [62]. Practically speaking, we found that as long as the chaotic states are avoided, the combs generally exhibit sufficient stability to feature low enough intensity noise to allow for their effective use in these applications.

The generated comb served as the multi-wavelength source for the TTDL and since the comb spectrum was stable, a waveshaper could be used to shape the comb lines in order to generate desired channel weights. The optical power difference between the comb lines could be compensated for via multi-stage spectral shaping combined with optical amplification [20] to meet practical systems' requirements. Looking to the future, high-conversion-efficiency Kerr combs generated by dark pulses [63] may provide an attractive solution to produce appropriately shaped combs with much higher energy efficiency. 
As Fig. 1 shows, the modulated signal after the MZM (EOSPACE) was propagated through $\sim 2.122-\mathrm{km}$ of single mode fibre with a dispersion of $\sim 17.4 \mathrm{ps} /(\mathrm{nm} \nabla \mathrm{km})$, corresponding to a time delay of $\sim 14.8 \mathrm{ps}$ between adjacent wavelength channels. We subsequently employed a Waveshaper (Finisar 4000s) as a wavelength division multiplexer to separate these channels, and then convert them back into the RF domain for PAA applications, thus achieving a high-channel count (up to 81 around the pump wavelength) TTDL with a compact structure.

The RF phase response was characterized by a vector network analyser (Anritsu 37369A), Fig. 3(a)), in which the channel at the central pump wavelength (channel 40) was set as the reference. The time delays corresponding to the measured phase slopes are shown in Fig. 3(b), in which a time delay step of $\sim 14.8$ $\mathrm{ps} /$ channel can be observed. To evaluate the uniformity of the delay steps between adjacent channels, we fit the measured delays with a second-order polynomial function

$$
\tau(\mu)=p_{0}+p_{1} \mu+p_{2} \mu^{2}
$$

where $\mu=1,2,3, \ldots, 81$, is the channel number, and $p_{0}, p_{1}, p_{2}$ are the fit coefficients. As Eq. 4 shows, uniform delay steps correspond to linear $t(\mu)$, while the fit curves $\left(p_{0}=6.7687, p_{1}=14.8, p_{2}=0.011878\right)$ shown in Fig. $3(b)$ indicate the appearance of small second-order terms arising from the third-order dispersion (TOD), thus introducing small delay errors in the TTDL (as extracted in the inset). Deviation in the comb wavelength spacing is extremely small $(<1 \mathrm{MHz})$, and so any delay errors induced by nonequidistance of the comb lines $\left(<10^{-3} \mathrm{fs} / \mathrm{step}\right)$ is negligible.

To investigate key performance parameters of the PAA driven by the micro-comb based TTDL, we calculated the array factors (AFs). Since many factors contribute to the performance of the PAA, such as delay errors, imprecision in channel weights, the number of radiating elements and rapid RF frequency variations, we adopted control variables during the investigation and calculated the AFs by varying only one parameter at a time.

In Fig. 3(c), the AFs are calculated with, and without, delay errors (uniform weights, $\lambda_{R F}=2.5 \mathrm{~cm}, d_{P A A}=$ $\left.\lambda_{R F} / 2, M=81, m=1\right)$. As can be seen, the delay errors induced by TOD shift the beam steering angle by $1.54^{\circ}$, and so this must be compensated for when designing the PAA system. We note that TOD compensation in fiber has been widely investigated using many approaches, and in our case this can easily be achieved by programming the phase characteristics of the waveshaper.

In Fig. 3(d), the AFs are calculated with generated weights (inset, where the weights of 5 comb lines are supressed for the calculation) and uniform weights ( $\lambda R F=2.5 \mathrm{~cm}, d P A A=\lambda R F / 2, M=81, m=1$, $\mathrm{T}=14.8 \mathrm{ps})$. Both of the AFs calculated this way yield different beam patterns that are each useful for specific applications. We note that arbitrary beam patterns can be achieved by shaping the channel weights, as we previously reported $[48,49]$. 
In Fig. 3 (e), AFs are calculated as as $M$ increases from 4 to 81 (uniform weights, $\lambda_{R F}=2.5 \mathrm{~cm}, d_{P A A}=\lambda_{R F}$ / $2, m=1, T=14.8 \mathrm{ps})$, where we see that the corresponding $3 \mathrm{~dB}$ beamwidth $\theta_{3 d B}$ significantly decreases from $26.8^{\circ}$ to $1.2^{\circ}$, matching well with the prediction $\theta_{3 d B}=102 / M$, as shown in Fig. 3(f). Compared with previous results based on a $200 \mathrm{GHz}-\mathrm{FSR}$ Kerr comb [49], the much larger channel number provided by the 49GHz-FSR Kerr comb significantly improves beam resolution for the PAA by more than a factor of four.

To achieve a tunable beam steering angle, every $m_{t h}(m=1,2,3, \ldots)$ wavelength of the TTDL can be selected by using the waveshaper. As well, the time delay $(\tau)$ between the radiating elements could be varied with a step size of $T$. As shown in Fig. 4(a), with 6 radiating elements $(M=6)$, as $m$ varies from 1 to 15 , a large tuning range from $-69.7^{\circ}$ to $72.9^{\circ}$ can be achieved. We note that tuning by selecting every $m_{t h}$ wavelength is practical in our case because of the large number of channels. The beamwidth $(\sim m)$ and steering angle tuning range $(\sim 1 / m)$ represent a trade-off in practical systems. To reflect the relationships between those parameters, in Fig. $4(\mathrm{~b}-\mathrm{e})$, we present AFs of PAAs with varying $m$ and $M$, where $M=$ [Channel Number $/ m$ ]. Considering the practical requirements for beam steering, $M$ can be set to 3 at least, and thus for the 21-channel PAA based on the 200GHz-FSR Kerr comb [49], $m$ could reach 7 at most, while for the 81-channel PAA based on the $49 \mathrm{GHz}$-FSR Kerr comb, $m$ could reach as much as 27. Fig. $4(\mathrm{~b}-\mathrm{c})$ shows the corresponding $M, \theta_{3 d B}$ and $\theta_{0}$ as a function of $m$. As can be seen, on the one hand, the $49 \mathrm{GHz}$ FSR Kerr comb enables a much larger $M$ as $m$ varies, thus leading to a significantly smaller $\theta_{3 d B}$ and greatly enhanced angular resolution (Fig. 4(d)). On the other hand, finer tuning steps (from $1.0^{\circ}$ to $14.7^{\circ}$ ) as well as a larger tuning range $\left(142.7^{\circ}\right)$ of the beam steering angle $\theta_{0}$ are available due to the larger $m$ (Fig. 4(e)).

Given the practical requirements in beamwidth and steering angle tuning range, optimized sets of $m$ and $M$ can be clearly identified from these calculations. Further, the beam steering angle can be improved in terms of both tuning step and tuning range by employing dispersion tunable media $[32,34]$ in addition to varying the number of TTDL channels.

Moreover, the PAA can also achieve a wide instantaneous RF bandwidth without beam squint (variation in beam steering angle with RF frequency). Based on the proposed TTDL, as indicated in Fig. 4(f), the beam steering angle $\theta_{O}$ remains $20.7^{\circ}$ while the RF frequency varies from $2 \mathrm{GHz}$ to $17 \mathrm{GHz}$ (with uniform weights, $\left.d_{P A A}=1.25 \mathrm{~cm}, m=1, M=81, T=14.8 \mathrm{ps}\right)$. As a result, due to our large number of channels, the PAA features greatly enhanced angular resolution, large instantaneous bandwidth, and a wide tunable range of the beam steering angle.

This approach is applicable to a wide range of wavelengths even outside the telecom band to the mid IR. [64-70] Soliton crystal microcombs have been extremely successful at a wide range of microwave and RF functions. [71-118]

\section{Conclusion}


We demonstrate a high channel count (81 over the C-band) TTDL based on an integrated optical frequency comb source. A broadband Kerr comb with a large number of comb lines was generated by an on-chip MRR with an FSR of $49 \mathrm{GHz}$, which was employed as a high-quality multi-wavelength source for the TTDL. Compared with traditional approaches, the size, complexity and, ultimately, the cost of the system can be greatly reduced. The large channel number of the TTDL resulted in a PAA with a calculated high angular resolution and wide tuning range of the beam steering angle. The enhancement in performance matches well with theory, confirming the feasibility of our approach as a promising solution towards implementing highly reconfigurable TTDLs for microwave photonic signal processing functions.

\section{Declarations}

\section{Competing interests:}

The authors declare no competing interests.

\section{References}

1. J. Capmany, and D. Novak, "Microwave photonics combines two worlds," Nat. Photonics 1, 319-330 (2007).

2. M. Ferrera, C. Reimer, A. Pasquazi, M. Peccianti, M. Clerici, L. Caspani, S. T. Chu, B. E. Little, R. Morandotti, and D. J. Moss, "CMOS compatible integrated all-optical radio frequency spectrum analyzer," Opt. Express 22, 21488-21498 (2014).

3. B. Corcoran, T. D. Vo, M. D. Pelusi, C. Monat, D. X. Xu, A. Densmore, R. B. Ma, S. Janz, D. J. Moss, and B. J. Eggleton, "Silicon nanowire based radio-frequency spectrum analyzer," Opt. Express 18, 2019020200 (2010).

4. M. Pelusi, F. Luan, T. D. Vo, M. R. E. Lamont, S. J. Madden, D. A. Bulla, D. Y. Choi, B. Luther-Davies, and B. J. Eggleton, "Photonic-chip-based radio-frequency spectrum analyser with terahertz bandwidth," Nat. Photonics 3, 139-143 (2009).

5. A. Pasquazi, M. Peccianti, B. E. Little, S. T. Chu, D. J. Moss, and R. Morandotti, “Stable, dual mode, high repetition rate mode-locked laser based on a microring resonator," Opt. Express 20, 2735527362 (2012).

6. J. P. Yao, “Microwave photonics," IEEE J. Lightwave Technol. 27, 314-335 (2009).

7. R. C. Williamson, and R. D. Esman, "RF photonics," IEEE J. Lightwave Technol. 26, 1145-1153 (2008).

8. A. J. Seeds, “Microwave photonics," IEEE T. Microw. Theory 50, 877-887 (2002).

9. K. Xu, R. X. Wang, Y. T. Dai, F. F. Yin, J. Q. Li, Y. F. Ji, and J. T. Lin, "Microwave photonics: radio-overfiber links, systems, and applications [Invited]," Photonics Res. 2, B54-B63 (2014).

10. R. A. Minasian, "Ultra-wideband and adaptive photonic signal processing of microwave signals," IEEE J. Quantum Elect. 52, 0600813 (2016). 
11. J. Capmany, B. Ortega, and D. Pastor, "A tutorial on microwave photonic filters," J. Lightwave Technol. 24, 201-229 (2006).

12. T. G. Nguyen, M. Shoeiby, S. T. Chu, B. E. Little, R. Morandotti, A. Mitchell, and D. J. Moss, "Integrated frequency comb source based Hilbert transformer for wideband microwave photonic phase analysis," Opt. Express 23, 22087-22097 (2015).

13. J. Wu, X. Xu, T. G. Nguyen, S. T. Chu, B. E. Little, A. Mitchell, R. Morandotti, and D. J. Moss, "Harnessing optical micro-combs for microwave photonics," arXiv: 1710.08611.

14. A. Malacarne, R. Ashrafi, Y. Park, and J. Azana, "Reconfigurable optical differential phase-shift-keying pattern recognition based on incoherent photonic processing," Opt. Lett. 36, 4290-4292 (2011).

15. Y. Park, M. H. Asghari, R. Helsten, and J. Azana, "Implementation of broadband microwave arbitraryorder time differential operators using a reconfigurable incoherent photonic processor," IEEE Photonics J. 2, 1040-1050 (2010).

16. J. Azana, C. Madsen, K. Takiguchi, and G. Cincotti, “Guest editorial - optical signal processing," J. Lightwave Technol. 24, 2484-2486 (2006).

17. S. Mansoori, and A. Mitchell, "RF transversal filter using an AOTF," IEEE Photonic Tech. L. 16, 879881 (2004).

18. X. Q. Zhu, F. Y. Chen, H. F. Peng, and Z. Y. Chen, "Novel programmable microwave photonic filter with arbitrary filtering shape and linear phase," Opt. Express 25, 9232-9243 (2017).

19. A. Ortigosa-Blanch, J. Mora, J. Capmany, B. Ortega, and D. Pastor, "Tunable radio-frequency photonic filter based on an actively mode-locked fiber laser," Opt. Lett. 31, 709-711 (2006).

20. X. X. Xue, Y. Xuan, H. J. Kim, J. Wang, D. E. Leaird, M. H. Qi, and A. M. Weiner, "Programmable singlebandpass photonic RF filter based on Kerr comb from a micro-ring," J. Lightwave Technol. 32, 35573565 (2014).

21. E. Hamidi, D. E. Leaird, and A. M. Weiner, "Tunable programmable microwave photonic filters based on an optical frequency comb," IEEE T. Microw. Theory 58, 3269-3278 (2010).

22. X. W. Ye, F. Z. Zhang, and S. L. Pan, "Optical true time delay unit for multi-beamforming," Opt. Express 23, 10002-10008 (2015).

23. D. H. Yang, and W. P. Lin, "Phased-array beam steering using optical true time delay technique," Opt. Commun. 350, 90-96 (2015).

24. Y. Q. Liu, J. P. Yao, and J. L. Yang, “Wideband true-time-delay unit for phased array beamforming using discrete-chirped fiber grating prism," Opt. Commun. 207, 177-187 (2002).

25. J. L. Cruz, B. Ortega, M. V. Andres, B. Gimeno, D. Pastor, J. Capmany, and L. Dong, "Chirped fibre Bragg gratings for phased-array antennas," Electron. Lett. 33, 545-546 (1997).

26. S. Chin, L. Thevenaz, J. Sancho, S. Sales, J. Capmany, P. Berger, J. Bourderionnet, and D. Dolfi, "Broadband true time delay for microwave signal processing, using slow light based on stimulated Brillouin scattering in optical fibers," Opt. Express 18, 22599-22613 (2010). 
27. K. Y. Song, M. G. Herraez, and L. Thevenaz, "Observation of pulse delaying and advancement in optical fibers using stimulated Brillouin scattering," Opt. Express 13, 82-88 (2005).

28. P. A. Morton, and J. B. Khurgin, "Microwave photonic delay line with separate tuning of the optical carrier," IEEE Photonic Tech. L. 21, 1686-1688 (2009).

29. O. F. Yilmaz, L. Yaron, S. Khaleghi, M. R. Chitgarha, M. Tur, and A. Willner, "True time delays using conversion/dispersion with flat magnitude response for wideband analog RF signals," Opt. Express 20, 8219-8227 (2012).

30. J. Mork, R. Kjaer, M. van der Poel, and K. Yvind, "Slow light in a semiconductor waveguide at gigahertz frequencies," Opt. Express 13, 8136-8145 (2005).

31. H. Su, P. Kondratko, and S. L. Chuang, "Variable optical delay using population oscillation and fourwave-mixing in semiconductor optical amplifiers," Opt. Express 14, 4800-4807 (2006).

32. J. J. Zhang, and J. P. Yao, "Photonic true-time delay beamforming using a switch-controlled wavelength-dependent recirculating loop," J. Lightwave Technol. 34, 3923-3929 (2016).

33. L. H. Zhang, M. Li, N. N. Shi, X. Y. Zhu, S. Q. Sun, J. Tang, W. Li, and N. H. Zhu, "Photonic true time delay beamforming technique with ultra-fast beam scanning," Opt. Express 25, 14524-14532 (2017).

34. Y. Q. Liu, J. L. Yang, and J. P. Yao, "Continuous true-time-delay beamforming for phased array antenna using a tunable chirped fiber grating delay line," IEEE Photonic Tech. L. 14, 1172-1174 (2002).

35. R. Wu, V. R. Supradeepa, C. M. Long, D. E. Leaird, and A. M. Weiner, "Generation of very flat optical frequency combs from continuous-wave lasers using cascaded intensity and phase modulators driven by tailored radio frequency waveforms," Opt. Lett. 35, 3234-3236 (2010).

36. J. Dai, X. Y. Xu, Z. L. Wu, Y. T. Dai, F. F. Yin, Y. Zhou, J. Q. Li, and K. Xu, “Self-oscillating optical frequency comb generator based on an optoelectronic oscillator employing cascaded modulators," Opt. Express 23, 30014-30019 (2015).

37. C. H. Chen, C. He, D. Zhu, R. H. Guo, F. Z. Zhang, and S. L. Pan, "Generation of a flat optical frequency comb based on a cascaded polarization modulator and phase modulator," Opt. Lett. 38, 3137-3139 (2013).

38. R. Wu, V. Torres-Company, D. E. Leaird, and A. M. Weiner, "Supercontinuum-based 10-GHz flat-topped optical frequency comb generation," Opt. Express 21, 6045-6052 (2013).

39. A. J. Metcalf, V. Torres-Company, D. E. Leaird, and A. M. Weiner, "High-power broadly tunable electrooptic frequency comb generator," IEEE J. Sel. Top Quant. 19, 231 (2013).

40. W. Z. Li, and J. P. Yao, "Optical frequency comb generation based on repeated frequency shifting using two Mach-Zehnder modulators and an asymmetric Mach-Zehnder interferometer," Opt. Express 17, 23712-23718 (2009).

41. T. Saitoh, M. Kourogi, and M. Ohtsu, "A waveguide-type optical-frequency comb generator," IEEE Photonic Tech. L. 7, 197-199 (1995). 
42. L. Razzari, D. Duchesne, M. Ferrera, R. Morandotti, S. Chu, B. E. Little, and D. J. Moss, “CMOScompatible integrated optical hyper-parametric oscillator," Nat. Photonics 4, 41-45 (2010).

43. D. J. Moss, R. Morandotti, A. L. Gaeta, and M. Lipson, "New CMOS-compatible platforms based on silicon nitride and Hydex for nonlinear optics," Nat. Photonics 7, 597-607 (2013).

44. A. Pasquazi, M. Pecciantia, L. Razzari, R. Morandotti, D. J. Moss, S. Coen, M. Erkintalo, T. Hansson, S. Wabnitz, P. Del Haye, and A. M. Weiner, "Micro-Combs: A Novel Generation of Optical Sources”, Physics Reports (2017). DOI:10.1016/j.physrep.2017.08.004.

45. M. Peccianti, M. Ferrera, L. Razzari, R. Morandotti, B. E. Little, S. T. Chu, D. J. Moss, “Sub-picosecond optical pulse compression via an integrated nonlinear chirper", Optics Express 18, 7625-7633 (2010).

46. D. Duchesne, M. Peccianti, M. Lamont, M. Ferrera, L. Razzari, F. Légaré, R. Morandotti, S. Chu, B. E. Little, D. J. Moss, "Supercontinuum generation in a high index doped silica glass spiral waveguide", Opt. Express 18, 923-930 (2010).

47. A. Pasquazi, Y. Park, J. Azaña, F. Légaré, R. Morandotti, B. E. Little, S. T. Chu, D. J. Moss, “Efficient wavelength conversion and net parametric gain via four wave mixing in a high index doped silica waveguide", Opt. Express 18, 7634-7641(2010).

48. X. Xu, J. Wu, M. Shoeiby, T. G. Nguyen, S. T. Chu, B. E. Little, R. Morandotti, A. Mitchell, and D. J. Moss, "Microwave photonic all-optical differentiator based on an integrated frequency comb source", Applied Physics Letters (APL) Photonics 2, 096104 (2017).

49. X. Xu, J. Wu, T. G. Nguyen, T. Moein, S. T. Chu, B. E. Little, R. Morandotti, A. Mitchell, and D. J. Moss, "Advanced RF and microwave functions based on an integrated optical frequency comb source," Opt. Express 26, (3) 2569-2583 (2018).

50. J. Wu, T. Moein, X. Xu, G. Ren, A. Mitchell, and D. J. Moss, “Micro-ring resonator quality factor enhancement via an integrated Fabry-Perot cavity”, Applied Physics Letters (APL) Photonics 2, 056103 (2017).

51. W. Liang, D. Eliyahu, V. S. Ilchenko, A. A. Savchenkov, A. B. Matsko, D. Seidel, and L. Maleki, “High spectral purity Kerr frequency comb radio frequency photonic oscillator," Nat. Commun. 6, 7957 (2015).

52. A. R. Johnson, Y. Okawachi, J. S. Levy, J. Cardenas, K. Saha, M. Lipson, and A. L. Gaeta, “Chip-based frequency combs with sub-100 GHz repetition rates," Opt. Lett. 37, 875-877 (2012).

53. W. Q. Wang, S. T. Chu, B. E. Little, A. Pasquazi, Y. S. Wang, L. R. Wang, W. F. Zhang, L. Wang, X. H. Hu, G. X. Wang, H. Hu, Y. L. Su, F. T. Li, Y. S. Liu, and W. Zhao, “Dual-pump Kerr micro-cavity optical frequency comb with varying FSR spacing," Sci. Rep. 6, 28501 (2016).

54. A. Strain, and M. Sorel, "Design and fabrication of integrated chirped Bragg gratings for on-chip dispersion control," IEEE J. Quantum Elect. 46, 774-782 (2010).

55. E. Sahin, K. J. A. Ooi, C. E. Png, and D. T. H. Tan, “Large, scalable dispersion engineering using cladding-modulated Bragg gratings on a silicon chip," Appl. Phys. Lett. 110, 161113 (2017). 
56. B. Stern, X. C. Ji, A. Dutt, and M. Lipson, "Compact narrow-linewidth integrated laser based on a lowloss silicon nitride ring resonator," Opt. Lett. 42, 4541-4544 (2017).

57. M. Longbrake, "True time-delay beamsteering for radar," in Aerospace and Electronics Conference (NAECON), 2012 IEEE National (IEEE, 2012), pp. 246-249.

58. M. I. Skolnik, Introduction to Radar Systems, 3rd ed. New York, NY: McGraw-Hill, 2001.

59. T. Herr, K. Hartinger, J. Riemensberger, C. Y. Wang, E. Gavartin, R. Holzwarth, M. L. Gorodetsky, and T. J. Kippenberg, "Universal formation dynamics and noise of Kerr-frequency combs in microresonators," Nat. Photonics 6, 480-487 (2012).

60. Y. K. Chembo, and C. R. Menyuk, “Spatiotemporal Lugiato-Lefever formalism for Kerr-comb generation in whispering-gallery-mode resonators," Phys. Rev. A 87, 053852 (2013).

61. S. Coen, H. G. Randle, T. Sylvestre, and M. Erkintalo, "Modeling of octave-spanning Kerr frequency combs using a generalized mean-field Lugiato-Lefever model," Opt. Lett. 38, 37-39 (2013).

62. D. C. Cole, E. S. Lamb, P. Del'Haye, S. A. Diddams, and S. B. Papp, "Soliton crystals in Kerr resonators," Nat. Photonics 11, 671-676 (2017).

63. X. X. Xue, Y. Xuan, Y. Liu, P. H. Wang, S. Chen, J. Wang, D. E. Leaird, M. H. Qi, and A. M. Weiner, “Modelocked dark pulse Kerr combs in normal-dispersion microresonators," Nat. Photonics 9, 594-600 (2015).

64. A. Della Torre et al., "Mid-infrared supercontinuum generation in a low-loss germanium-on-silicon waveguide", APL Photonics Vol. 6, 016102 (2021); doi: 10.1063/5.0033070.

65. M. Sinobad, et al., "Mid-infrared supercontinuum generation in silicon-germanium all-normal dispersion waveguides", Optics Letters, Vol. 45 (18), 5008-5011 (2020). DOI: 10.1364/OL.402159.

66. M. Sinobad et al., "High coherence at $f$ and $2 f$ of a mid-infrared supercontinuum in a silicon germanium waveguide", IEEE Journal of Selected Topics in Quantum Electronics Vol. 26 (2) 8201008 (2020). DOI:10.1109/JSTQE.2019.2943358.

67. M. Sinobad et al., "Dispersion trimming for mid-infrared supercontinuum generation in a hybrid chalcogenide Si-Ge waveguide", Journal of the Optical Society of America B, Vol. 36 (2) A98-A104 (2019). DOI: 10.1364/JOSAB.36.000A98.

68. M. Sinobad et al., "High brightness mid-infrared octave spanning supercontinuum generation to 8.5 $\mu \mathrm{m}$ in chip-based Si-Ge waveguides", Optica, Vol. 5 (4) 360-366 (2018).

DOI:10.1364/OPTICA.5.000360.

69. L. Jin et al., Applied Physics Letters Photonics, Vol, 5 Article 056106, (2020). DOI:10.1063/5.0002941

70. L. Carletti et al., "Nonlinear optical properties of Si-Ge waveguides in the mid-infrared”, Optics Express Vol. 23 (7) 8261-8271 (2015).

71. Mengxi Tan, X. Xu, J. Wu, T. G. Nguyen, S. T. Chu, B. E. Little, R. Morandotti, A. Mitchell, and David J. Moss, "Photonic Radio Frequency Channelizers based on Kerr Optical Micro-combs", Journal of Semiconductors <background-color:\#FFCC66;bu>42</background-color:\#FFCC66;bu> (4), 041302 (2021). 
72. H.Bao, L.Olivieri, M.Rowley, S.T. Chu, B.E. Little, R.Morandotti, D.J. Moss, J.S.T. Gongora, M.Peccianti and A.Pasquazi, "Laser Cavity Solitons and Turing Patterns in Microresonator Filtered Lasers: properties and perspectives”, Paper No. LA203-5, Paper No. 11672-5, SPIE LASE, SPIE Photonics West, San Francisco CA March 6-11 (2021). DOI:10.1117/12.2576645

73. Mengxi Tan, X. Xu, J. Wu, A. Boes, T. G. Nguyen, S. T. Chu, B. E. Little, R. Morandotti, A. Mitchell, and David J. Moss, "Advanced microwave signal generation and processing with soliton crystal microcombs", or "Photonic convolutional accelerator and neural network in the Tera-OPs regime based on Kerr microcombs", Paper No. 11689-38, PW210-OE201-67, Integrated Optics: Devices, Materials, and Technologies XXV, SPIE Photonics West, San Francisco CA March 6-11 (2021). DOI: $10.1117 / 12.2584017$

74. Mengxi Tan, Bill Corcoran, Xingyuan Xu, Andrew Boes, Jiayang Wu, Thach Nguyen, Sai T. Chu, Brent E. Little, Roberto Morandotti, Arnan Mitchell, and David J. Moss, "Optical data transmission at 40 Terabits/s with a Kerr soliton crystal microcomb”, Paper No.11713-8, PW210-0E803-23, NextGeneration Optical Communication: Components, Sub-Systems, and Systems X, SPIE Photonics West, San Francisco CA March 6-11 (2021). DOI:10.1117/12.2584014

75. Mengxi Tan, X. Xu, J. Wu, A. Boes, T. G. Nguyen, S. T. Chu, B. E. Little, R. Morandotti, A. Mitchell, and David J. Moss, "RF and microwave photonic, fractional differentiation, integration, and Hilbert transforms based on Kerr micro-combs", Paper No. 11713-16, PW210-OE803-24, Next-Generation Optical Communication: Components, Sub-Systems, and Systems X, SPIE Photonics West, San Francisco CA March 6-11 (2021). DOI:10.1117/12.2584018

76. Mengxi Tan, X. Xu, J. Wu, A. Boes, T. G. Nguyen, S. T. Chu, B. E. Little, R. Morandotti, A. Mitchell, and David J. Moss, "Broadband photonic RF channelizer with 90 channels based on a soliton crystal microcomb", or "Photonic microwave and RF channelizers based on Kerr micro-combs", Paper No. 11685-22, PW210-OE106-49, Terahertz, RF, Millimeter, and Submillimeter-Wave Technology and Applications XIV, SPIE Photonics West, San Francisco CA March 6-11 (2021).

DOI:10.1117/12.2584015

77. X. Xu, M. Tan, J. Wu, S. T. Chu, B. E. Little, R. Morandotti, A. Mitchell, B. Corcoran, D. Hicks, and D. J. Moss, "Photonic perceptron based on a Kerr microcomb for scalable high speed optical neural networks", IEEE Topical Meeting on Microwave Photonics (MPW), pp. 220-224, Matsue, Japan, November 24-26, 2020. DOI: 10.23919/MWP48676.2020.9314409

78. Mengxi Tan, Bill Corcoran, Xingyuan Xu, Andrew Boes, Jiayang Wu, Thach Nguyen, S.T. Chu, B. E. Little, Roberto Morandotti, Arnan Mitchell, and David J. Moss, "Ultra-high bandwidth optical data transmission with a microcomb”, IEEE Topical Meeting on Microwave Photonics (MPW), pp. 78-82. Virtual Conf., Matsue, Japan, November 24-26, 2020. DOI: 10.23919/MWP48676.2020.9314476

79. M. Tan, X. Xu, J. Wu, R. Morandotti, A. Mitchell, and D. J. Moss, "RF and microwave high bandwidth signal processing based on Kerr Micro-combs", Advances in Physics X, VOL. 6, NO. 1, 1838946 (2020). DOI:10.1080/23746149.2020.1838946.

80. Mengxi Tan, Xingyuan Xu, Jiayang Wu, Thach G. Nguyen, Sai T. Chu, Brent E. Little, Roberto Morandotti, Arnan Mitchell, and David J. Moss, "Photonic Radio Frequency Channelizers based on 
Kerr Micro-combs and Integrated Micro-ring Resonators", JOSarXiv.202010.0002.

81. Mengxi Tan, Xingyuan Xu, David Moss “Tunable Broadband RF Photonic Fractional Hilbert Transformer Based on a Soliton Crystal Microcomb", Preprints, DOI:10.20944/preprints202104.0162.v1

82. Mengxi Tan, X. Xu, J. Wu, T. G. Nguyen, S. T. Chu, B. E. Little, R. Morandotti, A. Mitchell, and David J. Moss, "Orthogonally polarized Photonic Radio Frequency single sideband generation with integrated micro-ring resonators", Journal of Semiconductors < background-color:\#FFCC66;bu>42</backgroundcolor:\#FFCC66;bu> (4), 041305 (2021). DOI: 10.1088/1674-4926/42/4/041305.

83. Mengxi Tan, Xingyuan Xu, Jiayang Wu, David J. Moss, "High bandwidth temporal RF photonic signal processing with Kerr micro-combs: integration, fractional differentiation and Hilbert transforms", arXiv:2103.03674 [physics.app-ph] (2021).

84. D.J. Moss, "Optimization of Optical Filters based on Integrated Coupled Sagnac Loop Reflectors”, Research Square (2021). DOI: https://doi.org/10.21203/rs.3.rs-478204/v1

85. Mengxi Tan, Xingyuan Xu, David J. Moss, "Integral order photonic RF and microwave signal processors based on Kerr micro-combs", Research Square https://www.researchsquare.com/article/rs-441212/v1 (2021). DOI:10.21203/rs.3.rs-441212/v1.

86. D.J. Moss, "Integral Order RF and Microwave Photonic Based Signal Processors Using Kerr Soliton Crystal Micro-combs." OSF Preprints, 31 May 2021. DOI:10.31219/osf.io/fvzw6 (2021).

87. Mengxi Tan, Xingyuan Xu, Jiayang Wu, Bill Corcoran, Andreas Boes, Thach G. Nguyen, Sai T. Chu, Brent E. Little, Roberto Morandotti, Arthur Lowery, Arnan Mitchell, and David J. Moss, “Highly reconfigurable RF photonic fractional Hilbert transformer”, Journal of Lightwave Technology, Vol. 39, Early Access (2021). DOI:10.1109/JLT.2021.3101816.

88. Mengxi Tan, Xingyuan Xu, Jiayang Wu, Roberto Morandotti, Arnan Mitchell, and David J. Moss, "RF and microwave photonic temporal signal processing with Kerr micro-combs", Advances in Physics $\mathrm{X}$, VOL. 6, NO. 1, 1838946 (2021). DOI:10.1080/23746149.2020.1838946.

89. Y. Zhang, J. Wu, Y. Yang, Y. Qu, L. Jia, T. Moein, B. Jia, D.J. Moss, “Enhanced nonlinear optical figureof-merit at 1550nm for silicon nanowires integrated with graphene oxide layered films", arXiv:2004.08043 (2020).

90. Yuning Zhang, Jiayang Wu, Yang Qu, Linnan Jia, Baohua Jia, and David J. Moss, “Design and optimization of four-wave mixing in microring resonators integrated with 2D graphene oxide films", Journal of Lightwave Technology, Vol. 39 Early Access (2021). DOI:10.1109/JLT.2021.3101292.

91. J. Wu et al., "Nested configuration of silicon microring resonator with multiple coupling regimes," IEEE Photon. Technol. Lett., vol. 25, no. 6, pp. 580-583 (2013).

92. J. Wu, T. Moein, X. Xu, and D. J. Moss, "Advanced photonic filters based on cascaded Sagnac loop reflector resonators in silicon-on-insulator nanowires," APL Photonics, vol. 3, 046102 (2018).

93. J. Wu, T. Moein, X. Xu, G. H. Ren, A. Mitchell, and D. J. Moss, "Micro-ring resonator quality factor enhancement via an integrated Fabry-Perot cavity," APL Photonics, vol. 2, 056103 (2017). 
94. H. Arianfard, J. Wu, S. Juodkazis, and D. J. Moss, "Advanced Multi-Functional Integrated Photonic Filters Based on Coupled Sagnac Loop Reflectors", Journal of Lightwave Technology, Vol. 39, No.5, 1400-1408 (2021). DOI: 10.1109/JLT.2020.3037559.

95. Hamed Arianfard, Jiayang Wu, Saulius Juodkazis, David J. Moss, "Spectral Shaping Based on Integrated Coupled Sagnac Loop Reflectors Formed by a Self-Coupled Wire Waveguide", IEEE Photonics Technology Letters, Vol. 33 (13) 680-683 (2021). DOI:10.1109/LPT.2021.3088089.

96. Hamed Arianfard, Jiayang Wu, Saulius Juodkazis and David J. Moss, "Three Waveguide Coupled Sagnac Loop Reflectors for Advanced Spectral Engineering”, Journal of Lightwave Technology, Vol. 39 (11) 3478-3487 (2021). DOI: 10.1109/JLT.2021.3066256.

97. Yuning Zhang, Jiayang Wu, Yang Qu, Linnan Jia, Baohua Jia, and David J. Moss, “Optimizing the Kerr nonlinear optical performance of silicon waveguides integrated with 2D graphene oxide films", Journal of Lightwave Technology, Vol. 39 Issue: 14, 4671-4683 (2021). DOI:

10.1109/JLT.2021.3069733.

98. Piotr Roztocki, Benjamin MacLellan, Mehedi Islam, Christian Reimer, Bennet Fischer, Stefania Sciara, Robin Helsten, Yoann Jestin, Alfonso Cino, Sai T. Chu, Brent Little, David J. Moss, Michael Kues, Roberto Morandotti, "Arbitrary phase access for stable fiber interferometers", Laser and Photonics Reviews, Vol. 15 (4) 2000524 (2021). DOI: 10.1002/Ipor.202000524.

99. Yang Qu, Jiayang Wu, Yuning Zhang, Yao Liang, Baohua Jia, and David J. Moss, "Analysis of fourwave mixing in silicon nitride waveguides integrated with 2D layered graphene oxide films", Journal of Lightwave Technology Vol. 39 (9) 2902-2910 (2021). DOI: 10.1109/JLT.2021.3059721.

100. Jiayang Wu, Linnan Jia, Yuning Zhang, Yang Qu, Baohua Jia, and David J. Moss, “Graphene oxide: versatile films for flat optics to nonlinear photonic chips", Advanced Materials, Vol. 33 (3) 2006415, 1-29 (2021). DOI:10.1002/adma.202006415.

101. Hamed Arianfard, Jiayang Wu, Saulius Juodkazis and David J. Moss, "Advanced Multi-Functional Integrated Photonic Filters based on Coupled Sagnac Loop Reflectors", Journal of Lightwave Technology, Vol. 39, Issue: 5, 1400-1408 (2021). DOI:10.1109/JLT.2020.3037559.

102. Yang Qu, Jiayang Wu, Yunyi Yang, Yuning Zhang, Yao Liang, Houssein El Dirani, Romain Crochemore, Pierre Demongodin, Corrado Sciancalepore, Christian Grillet, Christelle Monat, Baohua Jia, and David J. Moss, “Enhanced nonlinear four-wave mixing in silicon nitride waveguides integrated with 2D layered graphene oxide films", Advanced Optical Materials, Vol. 8 (21) 2001048 (2020). DOI: 10.1002/adom.202001048.

103. Yuning Zhang, Yang Qu, Jiayang Wu, Linnan Jia, Yunyi Yang, Xingyuan Xu, Baohua Jia, and David J. Moss, "Enhanced Kerr nonlinearity and nonlinear figure of merit in silicon nanowires integrated with 2D graphene oxide films", ACS Applied Materials and Interfaces, Vol. 12 (29) 33094-33103 (2020). DOI:10.1021/acsami.0c07852

104. X. Xu, et al., "Orthogonally polarized RF optical single sideband generation and dual-channel equalization based on an integrated microring resonator," Journal of Lightwave Technology, vol. 36, no. 20, pp. 4808-4818. 2018. 
105. M. Tan et al, "Orthogonally polarized Photonic Radio Frequency single sideband generation with integrated micro-ring resonators", IOP Journal of Semiconductors, Vol. 42 (2021). DOI:10.13140/RG.2.2.17603.63523.

106. H. Bao, et al., Laser cavity-soliton microcombs, Nature Photonics, vol. 13, no. 6, pp. 384-389, Jun. 2019.

107. B. Corcoran, et al., "Ultra-dense optical data transmission over standard fiber with a single chip source", Nature Communications, vol. 11, Article:2568 (2020).

108. Kues, M. et al. Quantum optical microcombs. Nature Photonics 13, (3) 170-179 (2019). doi:10.1038/s41566-019-0363-0

109. P.Roztocki et al., "Complex quantum state generation and coherent control based on integrated frequency combs”, Journal of Lightwave Technology 37 (2) 338-347 (2019).

110. S. Sciara et al., "Generation and Processing of Complex Photon States with Quantum Frequency Combs", IEEE Photonics Technology Letters 31 (23) 1862-1865 (2019). DOI: 10.1109/LPT.2019.2944564.

111. L. Caspani, C. Reimer, M. Kues, et al., "Multifrequency sources of quantum correlated photon pairs on-chip: a path toward integrated Quantum Frequency Combs," Nanophotonics, vol. 5, no. 2, pp. 351-362, 2016.

112. C. Reimer et al., "Generation of multiphoton entangled quantum states by means of integrated frequency combs," Science, vol. 351, no. 6278, pp. 1176-1180, 2016.

113. M. Kues, et al., "On-chip generation of high-dimensional entangled quantum states and their coherent control", Nature, vol. 546, no. 7660, pp. 622-626, 2017.

114. P. Roztocki et al., "Practical system for the generation of pulsed quantum frequency combs," Optics Express, vol. 25, no.16, 18940-18949, 2017.

115. Y. Zhang, et al., "Induced photon correlations through superposition of two four-wave mixing processes in integrated cavities", Laser and Photonics Reviews, vol. 14, no. 7, pp. 2000128, 2020. DOI: $10.1002 /$ lpor.202000128

116. C. Reimer, et al.,"High-dimensional one-way quantum processing implemented on d-level cluster states", Nature Physics, vol. 15 (2) 148 (2019).

117. X. Xu et al, "Photonic perceptron based on a Kerr microcomb for scalable high speed optical neural networks", Laser and Photonics Reviews, vol. 14, no. 8, 2000070 (2020). DOI: 10.1002/Ipor.202000070.

118. X. Xu, et al., "11 TOPs photonic convolutional accelerator for optical neural networks", Nature 589, 44-51 (2021).

\section{Figures}




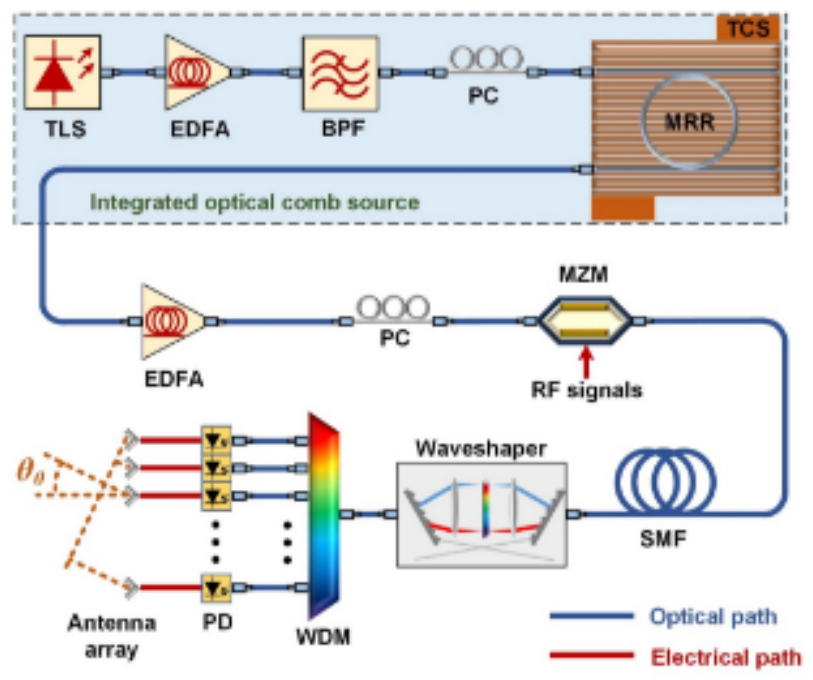

Fig. 1. Scheme of the proposed TTDL based on an integrated optical comb source. TLS: tumable laser source. EDFA: erbium-doped fibre amplifier. BPF: optical bandpass filter. PC: polarization controller. TCS: temperature controller stage. MZM: Mach-Zehnder modulator. SMF: single mode fibre. WDM: wavelength division multiplexer. PD: photodetector.

\section{Figure 1}

Scheme of the proposed TTDL based on an integrated optical comb source. TLS: tunable laser source. EDFA: erbium-doped fibre amplifier. BPF: optical bandpass filter. PC: polarization controller. TCS: temperature controller stage. MZM: Mach-Zehnder modulator. SMF: single mode fibre. WDM: wavelength division multiplexer. PD: photodetector.

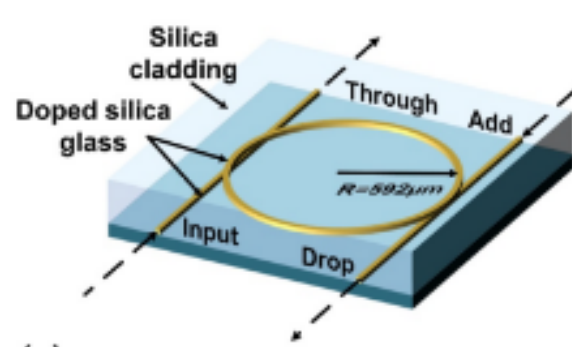

(a)

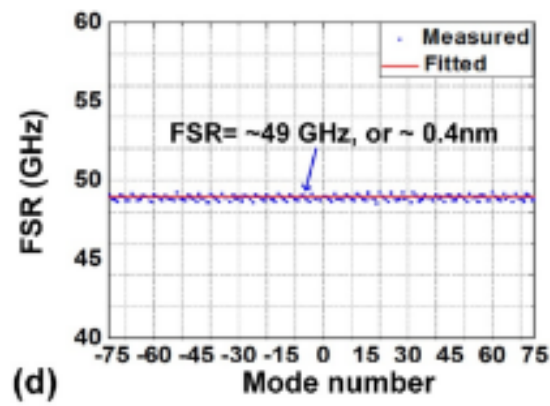

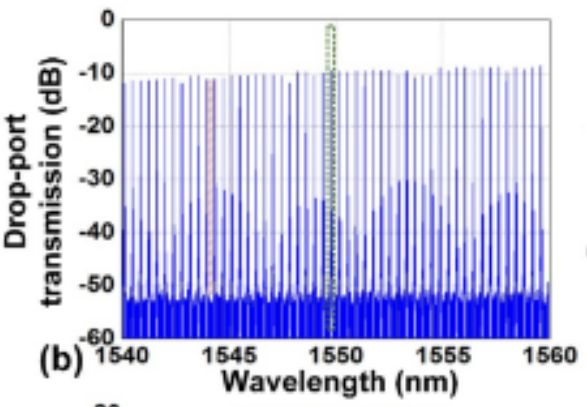

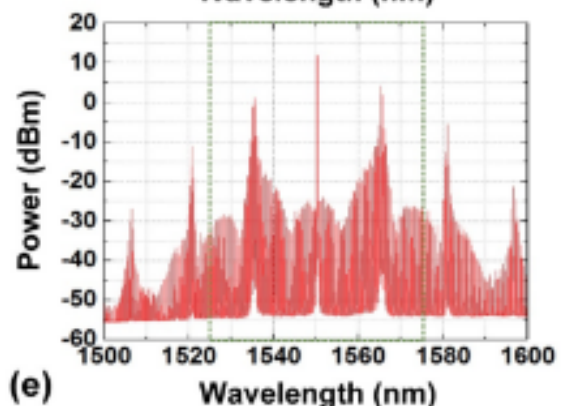

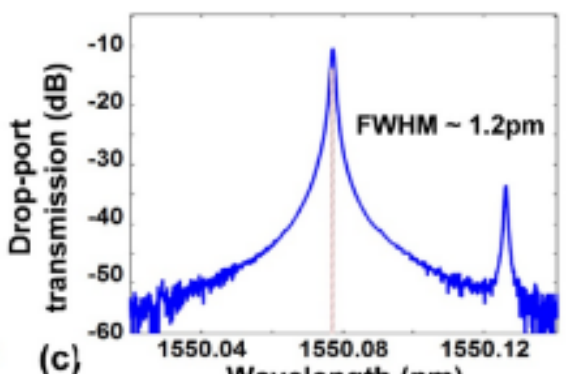

(c) Wavelength $(\mathrm{nm})$

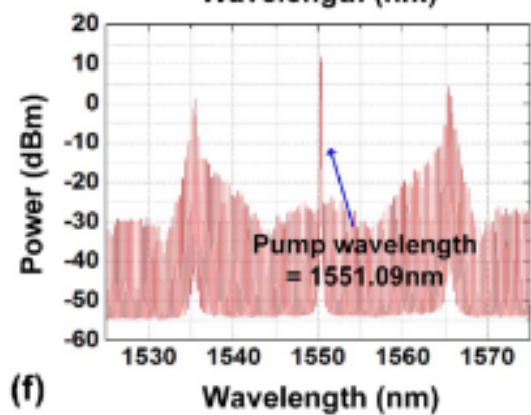

Fig. 2. (a) Schematic illustration of the MRR. Drop-port transmission spectrum of the on-chip MRR (b) with a span of $20 \mathrm{~nm}$, showing an FSR of $\sim 0.4 \mathrm{~nm}$, and (c) a resonance at $\sim 1550 \mathrm{~mm}$ with full width at half maximum (FWHM) of $\sim 1.2 \mathrm{pm}(\sim 150 \mathrm{MHz}$ ). (d) Measured and fitted FSR of the MRR. Optical spectrum of the generated Kerr comb with a span of (e) $100 \mathrm{~mm}$ and (f) $50 \mathrm{~mm}$. 
(a) Schematic illustration of the MRR. Drop-port transmission spectrum of the on-chip MRR (b) with a span of $20 \mathrm{~nm}$, showing an FSR of $\sim 0.4 \mathrm{~nm}$, and (c) a resonance at $\sim 1550 \mathrm{~nm}$ with full width at half maximum (FWHM) of $\sim 1.2 \mathrm{pm}(\sim 150 \mathrm{MHz})$. (d) Measured and fitted FSR of the MRR. Optical spectrum of the generated Kerr comb with a span of (e) $100 \mathrm{~nm}$ and (f) $50 \mathrm{~nm}$.
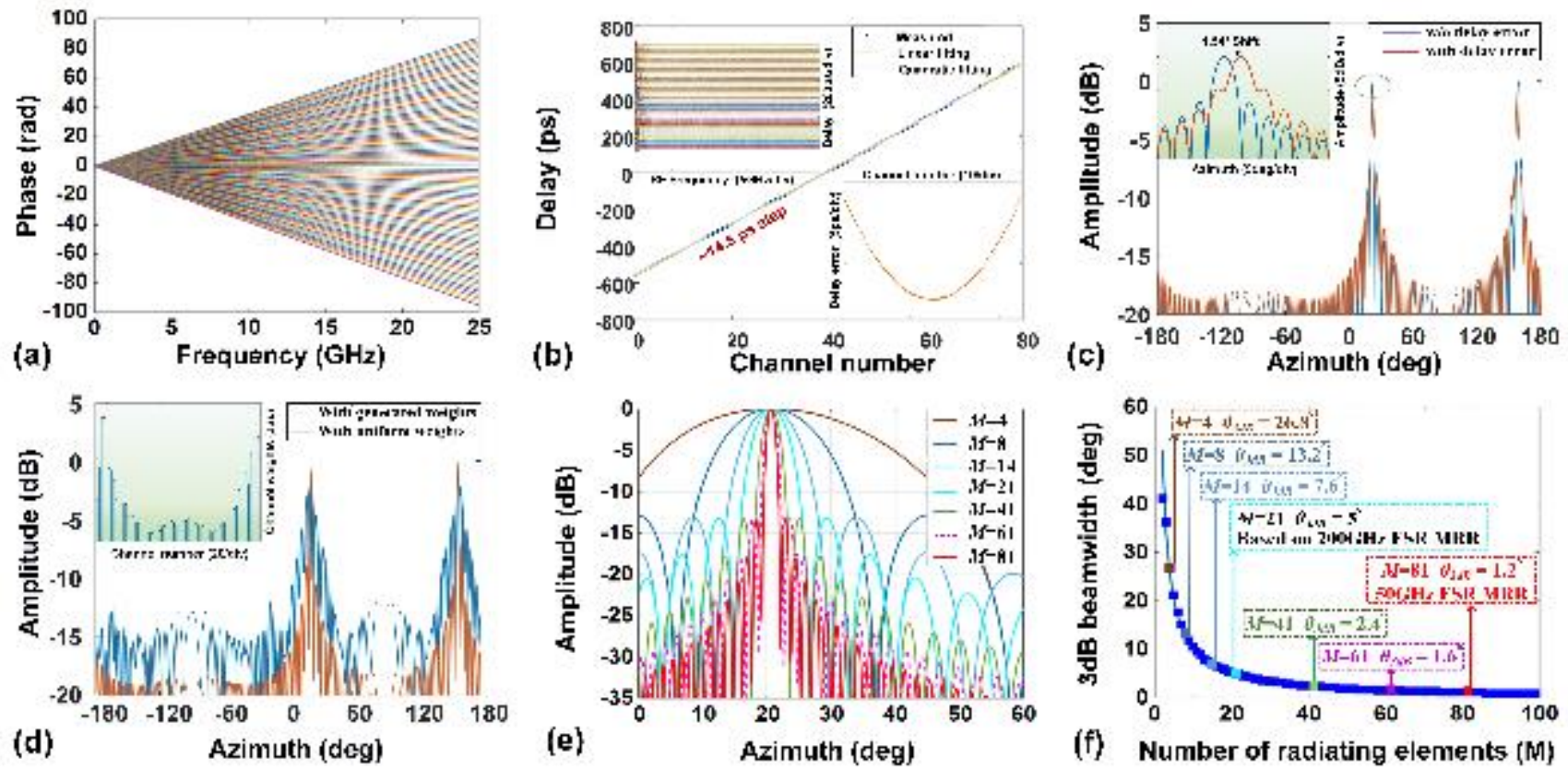

Fig. 3. (a) Measured RF phase response of the 81 -charnel TTDL and (b) corresponding time delays of each channel. The inset shows flat delays over a wide RF frequency range together with the extracted delay errors. (c) Calculated amay factors both with and without delay errors. (d) Calculated anry factors with generated weights and with uniform weights. (e) Calculated anray factors with $M$ varying from 4 to 81 . (f) Relationship between the number of radiating element $(M)$ and the $3 \mathrm{~dB}$ beamwidth $\left(\theta_{3 \mathrm{~s} B}\right)$.

\section{Figure 3}

(a) Measured RF phase response of the 81-channel TTDL and (b) corresponding time delays of each channel. The inset shows flat delays over a wide RF frequency range together with the extracted delay errors. (c) Calculated array factors both with and without delay errors. (d) Calculated array factors with generated weights and with uniform weights. (e) Calculated array factors with $M$ varying from 4 to 81 . (f) Relationship between the number of radiating elements $(M)$ and the $3 \mathrm{~dB}$ beamwidth $(\theta 3 \mathrm{~dB})$. 


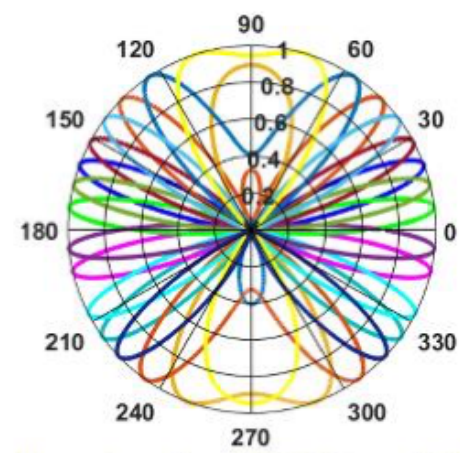

(a) Channel number $=81, M=6, m=1 \sim 15$ $\theta_{t \theta}=-69.7^{\circ} \sim 72.9^{\circ}$

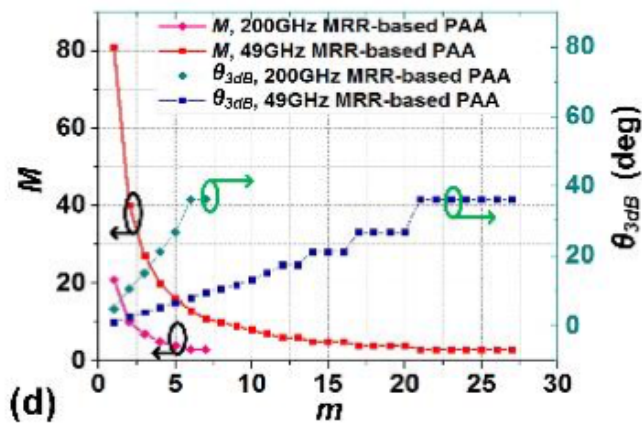

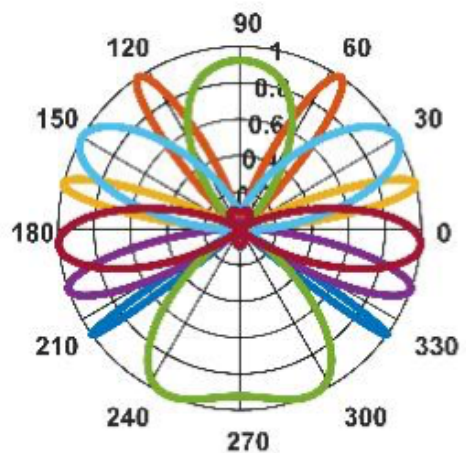

(b) Channel number $=21, m=1 \sim 7, \mathbf{M}=3 \sim 21$

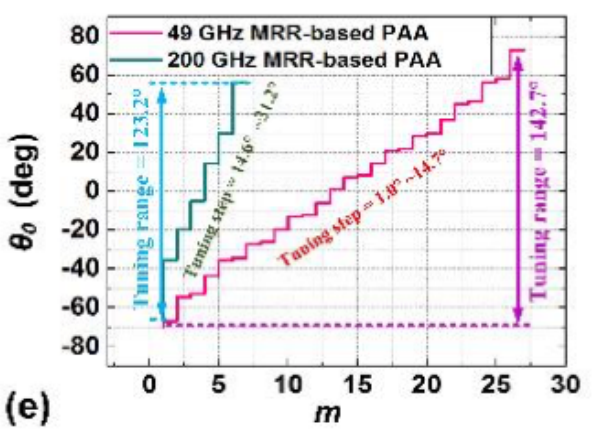

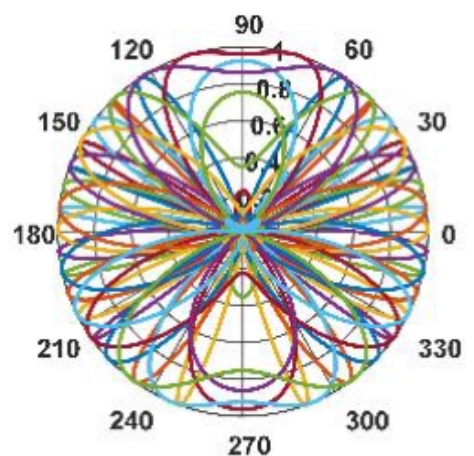

(C) Channel number $=81, m=1 \sim 27, M=3 \sim 81$

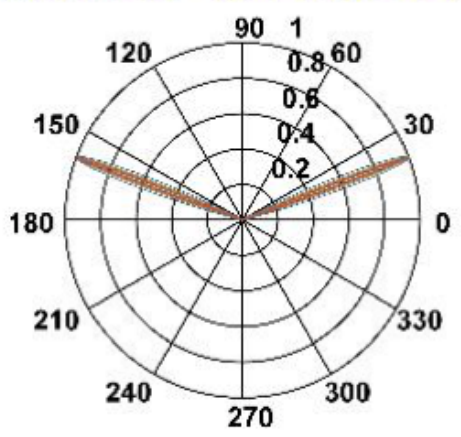

$\theta_{t h}=20.7^{\circ}, f_{k F}=2 \sim 17 \mathrm{GH} / \mathrm{z}$

Figure 4

(a) Calculated AFs of the PAA with $m$ varying from 1 to $15(M=6)$ based on the $49 \mathrm{GHz}-\mathrm{FSR}$ Kerr comb. (b) Calculated AFs of the PAA with m varying from 1 to 7 based on a 200GHz-FSR Kerr comb [49]. (c) Calculated AFs of the PAA with $m$ varying from 1 to 27 based on the $49 \mathrm{GHz}-F S R$ Kerr comb. (d) Number of radiating elements $(M)$ and the $3 \mathrm{~dB}$ beamwidth $(\theta 3 \mathrm{~dB})$ as a function of $\mathrm{m}$. (e) The beam steering angle $(\theta 0)$ as a function of $\mathrm{m}$. (f) Calculated AFs with RF frequencies varying from 2 to $17 \mathrm{GHz}$. 